\title{
Complementarity of Description and the Promise of Semiotics in Dealing with an Eluding Object
}

\section{A Commentary on Terrence W. Deacon's "How molecules became signs"}

\author{
Joanna Rączaszek-Leonardi ${ }^{1}[$
}

Received: 19 October 2021 / Accepted: 14 November 2021 / Published online: 15 December 2021

(c) The Author(s) 2021

\begin{abstract}
I emphasize the general character of the central claim made by Terrence Deacon about the necessity of complementary description of evolving cognitive systems. Next, I clarify and augment one of the claims made in the paper about the tools offered by information theory. Finally, I point to the need of further clarification of some central notions, which should help to make connections across discourses.
\end{abstract}

Keywords Semiotics - Complementarity of descriptions - Symbol emergence

Semantics $\cdot$ Interpretation

In his target paper Terry Deacon presents a possible scenario of "How molecules became signs". The title echoes (or rather acknowledges and presents a possible answer to) the famous question asked by Howard Hunt Pattee in 1969: "How does a molecule become a message?". The crucial contribution of the paper is that it shows compellingly that focus on the structure of a message itself will never tell us what it is about or what functions it has within a system. The statement may sound obvious but the pervasive tendency (in the biological, cognitive, neuro- and language sciences) to treat the structure of a message as the source of information "about" external events or "about" the system, and then searching for its grounding in such entities, warrants such deeper analysis and reflection.

In consequence, Deacon (2021) rightly points out that the information-theoretical notions (and measures), which were designed to characterize such internal structures for the purpose of message transmission (by Shannon and followers) will not account for its meaning. Concepts are needed that are able to give justice to the richness of historical processes of signification, of which any "messages" present

Joanna Rączaszek-Leonardi raczasze@psych.uw.edu.pl

1 Human Interactivity and Language Laboratory, Faculty of Psychology, University of Warsaw, Warsaw, Poland 
in living systems are a part. For that, Deacon uses the semiotic framework of CS Peirce, with its hierarchical structure of signification, which helps to understand, how complex messages may be scaffolded by the workings of iconic and indexical constraints. The applicability of such framework is illustrated with the basic model of an autogen, in which the primary "interpretive competence", namely distinguishing self from non-self, is progressively enriched by the ability to distinguish favourable environmental conditions (contextual autogen), and finally, leads to the template-mediated autogenesis, in which dynamical constraints find their embodiment in a copy-able structure. The latter gives an energetically cheaper, faster, and more reliable way of reproducing the self-recreating and self-maintaining dynamics than the low-probability process of recreating a constellation of dynamic constraints from scratch. This is the basis for the process of adaptation through evolution and selection.

As I basically agree with the proposed view and find the illustration convincing, in this short commentary I would like to do three things, with a goal of generalizing the conclusions and clarifying some points. First, I will emphasize the general character of the central claim made by Deacon, about the necessity of complementary description, and point to its immediate and wide consequences for the explanations in the cognitive and language sciences. Second, I would like to clarify and augment one of the claims pertaining to the tools offered by the information theory, regarding their usefulness and limitations for the study of cognition. Third, I would like to point to the necessity of further clarification of some central notions, which, due to their differential history of use in various domains are still, in my view, not sufficiently individuated to provide for connections across discourses.

\section{The Poverty of the Cognitive Sciences Toolbox}

Deacon's exposition makes it clear that the explanations of living and cognitive processes and systems need a complementarity of description. This thesis was posed already by von Neumann (1966) and developed by Pattee in the context of both biological information (Pattee, 1969, 1972), and the theory of cognition in general. Pattee's discussion and commentaries on the necessity of both formal (syntactic) descriptions of symbolic structures and descriptions of the dynamical processes constrained by such structures are already a classic in some circles (see e.g., Pattee, 1982, Pattee \& Rączaszek-Leonardi, 2012). By demonstrating this necessity, he has put in dialogue, instead of in opposition, two of the prevailing frameworks for cognitive psychology and cognitive science of the times: the information processing paradigm and ecological psychology/dynamical systems paradigm. Deacon (2021) powerfully illustrates the claims of von Neumann and Pattee with the model of an "autogen", in which the two descriptions are necessitated by two kinds of functioning of a physical structure, which arise in complementary processes: as a copy-able record and as an instruction for reconstruction. Only the first one can perhaps be understood in terms of coding, while the latter requires a description of how dynamics of a growing organism are constrained by these information structures. 
Here I wish to underscore that this general principle of complementarity, demonstrated by Deacon at the level of molecular biology, pertains to all levels of organization engaged in the process of adaptive complexification, and thus has profound consequences for other domains of cognitive and language sciences. The consequences include the necessity for enriching the theoretical toolbox with concepts that can better account for the processes of control of dynamics by replicable constraints, and enriching the researchers' toolbox with the methods and measurements that can better capture such processes.

One important implication of the proposed scenario for the emergence of autogen is that in the process of transferring a complex set of constraints from substrate to substrate, the "message", never becomes an abstract and immaterial "thing" - or a set of abstract symbols, which seem to be a staple substance of mind in a dualistic Cartesian picture. On the contrary: the process can be viewed, in some sense, as an opposition to what is usually meant by abstraction: it embodies, in a concrete physical structure, the complex dynamical and relational constraints that maintain an organism far from thermodynamic equilibrium. The physicality of the "generalized language system" in Pattee's work or of Deacon's templates, warrants their causal properties in harnessing the laws of physics by binding selected degrees of freedom to perform the work of re-creating and maintaining the system, while the harnessed dynamics warrants the ever present plasticity and creativity of the process. The substrate effectuating the harnessing may change but it never vanishes into an abstract realm. This brings us to the second point, that, being physical, and being engaged in physical processes these structures have properties, which are not easily captured by the standard information-theoretical tools that were designed to describe internal structure of one-dimensional messages for the purpose of their transmission.

Deacon's (2021) claim that the information-theoretical tools will not suffice to give justice to the richness of processes that are pertinent to the organization of living and cognitive processes is thus worth emphasizing and elaborating. It reveals the poverty of our toolbox as forgers of explanations in cognitive and language sciences. Since the significance of messages does not reduce to a simple mapping between a 'sign' and its 'referent' but rather is based on a hierarchical control infrastructure, which involves relations that are time-based and topological, methods that deal with transformations and internal organization of one-dimensional structures are not convenient to capture them. This has been pointed out in the past by the cognitive scientists (among others, Dreyfus, 1972, 1992) and ecological psychologists (Turvey \& Carello, 1981). However, the proposed - alternative - frameworks seem to chiefly concentrate on (and develop tools for) bringing back the dynamics and its organization into the picture, which is necessary but not sufficient. The comprehensive nature of Deacon's approach lies in capturing the relation between the structured messages and these dynamics. Deacon turns to the semiotic framework, which allows for complex, under-defined and dynamic objects (that elude simple mappings) and a variety of relations, of which some are spatial. It becomes evident that the semiotic explanation provides not just another set of labels for known processes, amenable to information-theoretical analysis, but is based in a richer ontology: more suitable to deal with causal structures, historical and long-range dependencies and 
topological relations. These are crucial when talking about constraining dynamics, but are smothered or ignored by the analyses which pertain to uni-dimensional symbol strings.

\section{The Usefulness and Limits of "Symbolic" Description}

Since Deacon's framework, following Pattee, gives due place for both the structured replicable templates and the dynamics of the reconstruction and functioning of an organism, it allows for a better understanding just where the information-theoretical tools are useful in describing and explaining cognition. Judging from their immense popularity, they indeed prove useful for some processes or stages of a cognitive process. Thus even though, as Deacon (2021) rightly claims, they do not reveal what the message is about, it is possible to read off of the message some properties of the dynamical processes in the control of which they are engaged. Deacon admits this in his paper in passing, but it is perhaps good to indicate, that substantial work still needs to be done to understand which properties of those processes, and at which level, can be captured this way. It will be helpful both for revealing the nature of the processes (amenability to certain kinds of description) and - most importantly - for indicating in a clearer way the limitations of the information-theoretical methods.

Thus, although I agree that "what makes information meaningful is interpretation", that is, the form and history of the interpretive process, and not the intrinsic properties of vehicles, the properties of the vehicles are correlated with the properties of the semantic processes, i.e., are unlikely to be completely arbitrary with respect to them. Thus the structure of constraints is informative about the processes they constrain, but, of course not in a direct-referential way. As Deacon says, the properties of sign vehicle do not 'determine' reference (being constraints they bias rather than determine the dynamics) but they are informative about some abstract properties of those processes.

This pertains to such properties as, for example, the order of the elements in a message, which often correlates with the order of events it is designed to control (which is clear in the case of vehicles that are time-ordered such as language, where one element precedes another and thus constraints exerted by the latter may "count on" those exerted by the former). Similarly, the range of variability of the message elements and the global complexity (though not necessarily its length) might correlate with the complexity of the processes that they evolved to constrain; the frequency of the elements of the message and/or relations among the elements might be correlated with the frequency of the control processes effectuated by these structures (or, in Deacon's terms, of the particular interpretive processes). Finally, and perhaps most importantly for the contemporary brain and cognitive sciences, similarities in message structures (and structures-in-time) might inform about the similarities of control and coordinative processes that those structures participate in. The sheer physical improbability of encountering such structures by chance, makes such similarity informative. Thus the usefulness of such measures as mutual information, correlations and lag-correlations among various signals generated by the cognitive systems, which in-form differences 
in the controlled processes, but can also inform the observer about such differences, especially of she knows the differences in the respective systems' boundary conditions.

To sum up, the similarity of intrinsic properties of messages can be informative about the relation between meaning-creating processes. Importantly, the information-theoretical tools will never touch upon directly on "what the message is about", because it lacks information on how those structures harness dynamical process, how they engage in the flow of energy to make sure that the work performed serves the organism. But they may be informative about relations among such meaningful processes - which is (relationally) meaningful.

\section{Towards a Common Language}

Finally, a short terminological note, which is motivated by the desire to establish a better contact among different discourses. Deacon's paper (2021) does an excellent job explaining in what respect we can talk about representations in cognitive and living systems. First, the interplay of dynamic constraints re-presents (over and over again) the favorable conditions for self-maintenance. Second, due to transferability of relations to a different substrate, "a molecular template literally re-presents the topology of the dynamical network of interactions that functions to re-present and re-produce itself". This is an important step in showing that talking about representing as an abstract mental entity standing for something concrete in the world is way too simplistic to be of use in the theory of cognition (see e.g., Freeman \& Skarda, 1990 for the analysis of the damage it usually does). The "interpretive competence" of an organism, which consists in its re-presenting its own boundary conditions cannot be usefully reduced to any simple mapping relation, which is usually evoked in the context of mental representations (Rączaszek-Leonardi, 2016).

However, other terms that Deacon uses in a different way than in the standard cognitive or linguistic discourses, were not so explicitly redefined. Although one can fully agree that what constraints do is "preserving a trace of past instantiations and past work", calling this "reference" might be misleading. The "trace of past instantiations and past work" is not what most linguists or cognitive scientists would call "a reference". Those traces, as Deacon writes, are "semiotic affordances" (a great term by the way), and as affordances, they do not "refer", but rather enable a favourable dynamics within a given environment. Similarly, the influence that such semiotic affordances exert, i.e., their preserving a trace of past instantiations and past work in order to re-present and reconstruct the self in various environments is not what a common passerby (nor cognitive scientist or linguist) would term "interpretation". Deacon's use of these terms follows Peirce, but it seems that such use is not prevalent enough to provide a stable link through these concepts to the literature in the cognitive and language sciences.

The term 'interpretation', it seems, can gain much clarity through the work by Deacon $(1997,2021)$, which gives it such a concrete physical-historical reading. In (folk) cognitive science it usually evokes an individualistic process of making sense in one's mind about external events, which - if any - has only a 
partial overlap with the above recording and reconstructive processes. The present approach allows to construct the relation of aboutness in terms of non-mentalistic constraining of processes of physical re-creation. It allows to ask in a novel and promising way: What parts of those processes can usefully be called mental? Are they necessarily individualistic? However, asking: "What form of molecular process is necessary and sufficient to interpret some property of a molecule as providing information about other molecular properties?" or "how a molecule can become about other molecules" might unnecessarily narrow down the meaning of the term "interpretation" and not be conducive to such clarification. It is not information about concrete molecular properties but rather complex constellations of constraints on dynamical processes that finds its way into structured constraints. Returning to Deacon's (2021) autogen illustration and taking a point of view of the autogen itself: no simple objects in the environment of the autogen will constitute the meaning of any messages exchanged between autogens (generationally or in interactions). Whatever messages are needed for, they are, as Deacon remarks, about the complex networks of dynamical constraints, and thus nonreducible to e.g., simple molecules or properties of molecules. The 'Object' of the aboutness remains under-defined and we should somehow learn to deal with that within the theory of cognition.

Funding The work on the paper was funded by NCN grant Opus 2018/29/B/HS1/00884.

\section{Declarations}

Conflicts of Interests/Competing Interests I declare no conflict of interest.

Open Access This article is licensed under a Creative Commons Attribution 4.0 International License, which permits use, sharing, adaptation, distribution and reproduction in any medium or format, as long as you give appropriate credit to the original author(s) and the source, provide a link to the Creative Commons licence, and indicate if changes were made. The images or other third party material in this article are included in the article's Creative Commons licence, unless indicated otherwise in a credit line to the material. If material is not included in the article's Creative Commons licence and your intended use is not permitted by statutory regulation or exceeds the permitted use, you will need to obtain permission directly from the copyright holder. To view a copy of this licence, visit http://creativecommons.org/licen ses/by/4.0/.

\section{References}

Deacon, T. W. (1997). The symbolic species: The co-evolution of language and the brain. W.W. Norton \& Company.

Deacon, T. W. (2021). How molecules became signs. Biosemiotics. Advance online publication. https://doi.org/10.1007/s12304-021-09453-9.

Dreyfus, H. L. (1972). What computers can't do: A critique of artificial reason. Harper \& Row.

Dreyfus, H. L. (1992). What computers still Can't do. MIT Press.

Freeman, W. J., \& Skarda, C. A. (1990). Representations: Who needs them? In J. L. McGaugh, N. M. Weinberger, \& G. Lynch (Eds.), Brain organization and memory (pp. 375-380). Oxford University Press. 
Pattee, H. H. (1969). How does a molecule become a message? In A. Lang (Ed.), Communication in development (Vol. 3, p. 1e16). Academic Press.

Pattee, H. H. (1972). Laws and constraints, symbols and languages. In C. H. Waddington (Ed.), Towards a theoretical biology 4, essays (p. 248e258). Edinburgh University Press.

Pattee, H. H. (1982). Cell psychology: An evolutionary approach to the symbol matter problem. Cognition and Brain Theory, 5(4), 325e341.

Pattee, H.H. \& Rączaszek-Leonardi, J. (2012). Laws, Language and Life. Springer.

Rączaszek-Leonardi, J. (2016). How does a word become a message? An illustration on a developmental timescale. New Ideas in Psychology, 42, 46-55. https://doi.org/10.1016/j.newideapsych. 2015.08.001.

Turvey, M. T., \& Carello, C. (1981). Cognition: The view from ecological realism. Cognition, 10(13), 313-321.

Von Neumann, J. J. (1966). Theory of self-reproducing automata. Edited and completed by a.W. Burks. University of Illinois Press.

Publisher's Note Springer Nature remains neutral with regard to jurisdictional claims in published maps and institutional affiliations. 